\title{
Interactive comment on "High-latitude crochet: solar flare-induced magnetic disturbance independent from low-latitude" by Masatoshi Yamauchi et al.
}

\section{Masatoshi Yamauchi et al.}

m.yamauchi@irf.se

Received and published: 22 August 2020

Thank you for your kind and constructive comments.

\#reviewer\# A crochet is a type of geomagnetic disturbance that is typically observed at low and middle latitudes following a solar flare. This paper describes characteristics of a new type of geomagnetic crochet at high latitudes $(65-75 \mathrm{~N})$. It is shown that the new crochet differs from ordinary ones at lower latitudes in terms of its intensity and duration. The new crochet is also shown to be different from previously reported crochets in the auroral and cusp regions. The paper contains new and exciting results that make a good addition to the understanding of the geomagnetic field. As such, I recommend 
this paper for publication. Below are my comments and suggestions that could further improve the quality of the paper.

Followings are answers to your specific questions/comment (\#1, 4, 5, 7, 8, 10, 12, 13, 15,16 , and 18), and these explanation are included in the revised manuscript.

\#comment 1.\# "Sq (ion) convection" (I.12,13,167,171,172,282), "Sq" (I.236) In my opinion, the term Sq should not be used when referring to quiet-day electric fields or currents at high latitudes. Sq electric fields and currents at middle and low latitudes are produced by the wind dynamo. At high latitudes, daily variations in electric fields and currents are not due to the wind dynamo but due to the magnetospheric convection, thus calling them Sq can be confusing. My suggestion is as follows:

I.12 Remove "Sq".

I.13 Replace "Sq" with "background".

I.167 Remove "Sq".

I.171 Replace "Sq" with "background".

I.172 Remove "Sq".

I.236 Replace "Sq" with "background".

I.282 Replace "Sq" with "background".

\#Answer\# I realized that we did not clearly differentiate Sq0 (tidal-driven only) and SqP (mixed with solar wind driven). When we subtract background level, it is "Sq0 + SqP" ("background" is a better name), but when we discuss "enhancement of Sq, it is Sq0 ("Sq" is the better name). We change wording respectively, with additional reference by Matsushita (1958).

Matsushita, S.: Interactions Between the lonosphere and the Magnetosphere for Sq and L Variations, Radio Sci., 6( 2), 279? 294,

Interactive

comment
Printer-friendly version

Discussion paper 
https://doi.org/10.1029/RS006i002p00279, 1971.

\#comment 4.\# I.70 "The other data are described" What are "the other data"?

ANGEOD

\#Answer\# It is non-essential data for this paper (ACE data, GOES energetic particle data) but readers may want to see. We explain explicitly.

Interactive

\#comment 5.\# I.126 "Equivalent ionospheric current" Please briefly describe how the comment baseline was determined. The baseline matters for equivalent currents.

\#Answer\# We believe that the reviewer is referring "baseline" to "quiet level" (a value without Sq), i.e., internal field. (We normally use "baseline" terminology to calibration of flux gate magnetometer data, but we think the reviewer does not mean that.) Yes, we remove this "quiet level" prior to the application of the SECS technique.

The "quiet level" is determined by assuming that majority of lesser disturbances occur at around this value. Derivation is achieved by a least-square-root approximation, where the square root emphasizes the small variations around this "quiet level", rather than larger disturbances (e.g., substorms). The derivation is made for each "target day" using 10 days of data centered on the day of interest. This avoids contamination of main field secular variation but still gives sufficient data to determine "quiet level". The uncertainty is estimated to be less than $10 \mathrm{nT}$. See. eg. Edvardsen et al. (2013) for more details.

Edvardsen, I., Hansen, T. L., Gjertsen, M., \& Wilson, H.: Improving the accuracy of directional wellbore surveying in the Norwegian Sea, Soc. Petrol. Eng. Drill. Complet., 28, https://doi.org/10.2118/159679-PA, 2013.

\#comment 7.\# I.164 "EISCAT VHF radar" What is the antenna direction? The Figure 5 caption says that the radar was looking northward with $30^{\circ}$ angle. Is this from the vertical or local magnetic field line, or something else?

\#Answer\# It is $30^{\circ}$ "elevation" which is the lowest allowed elevation.

Printer-friendly version

Discussion paper 
\#comment 8.\# Table 1 I do not understand this table. For example, for ASY, I see that a crochet was detected in 52 flare events; not detected in 5 events; and unclear in 6 events. Additionally, there were 5 events where a crochet was unclear because of substorm-related disturbances. But they do not add up to the total 73 events. " +5 " in the "yes" category is unexplained. Also, it is strange to see that the number of "substorm" is different for ASY, AU, and AL. Would not it be more straightforward if the table is created only for the 62 events which are not concurrent with a substorm?

\#Answer\# We are sorry for insufficient explanation. Five case at @Onset means simultaneous onsets of substorm and crochet. We change the way to show as 57 (5) instead of $52(+5)$. Substorm column means that substorm activity is to disturbed to isolate the crochet effect. Since substorm disturbance is stronger in $A U / A E$, it is more difficult to see it. If only ASY, substorm activity is mild enough to isolate more crochet. We add these explanations.

\#comment 10.\# I.192 "they are either auroral" Or what?

\#Answer\# "auroral crochet or this new high-latitude crochet." (When we copy-andpasted from word to Latex, copy was not complete).

\#comment 12.\# I.232 "if intensification of the Sq current is important, the new crochet might be the equinox phenomenon" This may be removed. Sq currents at middle and low latitudes exist not only during equinox but also during solstice.

\#Answer\# What we meant here is inter-hemispheric coupling of Sq current along the geomagnetic field. This is important for high-latitude. We add inter-hemispheric aspect in the explanation.

\#comment 13.\# I.236 "through the enhancement of both the ion/electron density and ion velocity" The enhancement of plasma density can be understood as a result of increased ionization during the solar flare, but how do the authors explain the enhancement of ion velocity (i.e., electric field)?

Interactive

comment 
\#Answer\# Yes it is obvious question. It is so obvious the we forgot to write this question. \#comment 15.\# I.249 "and traditional explanation of the trigger is IMF changes" I do not understand what was meant by this. Remove or rephrase.

\#Answer\# We add explanation. Although this substorm is most likely associated with the southward IMF during 18:29-18:57 UT at the Sun-Earth first Lagrange point L1, timing of the substorm onset and large AU compared to large AL suggests that crochet could triggered the onset.

\#comment 16.\# I.261 "on that day" Please clarify which day.

\#Answer\# 6 September, 2017 (same day as X9.3 flare)

\#comment 18.\# I.268 "4.4 Relation to space weather" This subsection, consisting of two sentences, can be removed. It does not add any new information or insight.

\#Answer\# A large sunspot may cause coincident occurrence of strong solar flare and large substorm triggered by the coronal mass ejection (CME). If the crochet mechanism can interact with a substorm and reinforce each other, and if the strong solar flare takes place within an hour after CME hits the Earth, we expect extremely strong ionospheric current and resultant ground induced currents (GIC) that are hazardous. I add this explanation.

All other comments (typo/expression/error) are amended as suggested

\#comment 2.\# I.1 "Solar flare-induced High latitude" "High" should be in the lower case.

\#comment 3.\# I.36 "it is simple called crochet" Replace "simple" with "simply".

\#comment 6.\# I.162 "daily neutral convection starting from subsolar region" This entire phrase can be replaced by "tidal winds".

\#comment 9.\# I.187 "There are about 10 events are during substorms" Insert "that" between "events" and "are". 
\#comment 11.\# I.208 "This suggest that AU signature is most likely caused by this crochet rather than auroral crochet." This is difficult to say without data from other LT. I suggest to replace "is most likely" with "could be". Also, replace "suggest" with "suggests".

\#comment 14.\# I.242 "Such a work also probably give some hints" Replace "give" with "gives".

\#comment 17.\# I.264 "mediation" "modulation"?

\#comment 19.\# Finally, please check the numbering of sections and subsections, which is currently as follows: ... ... Subsection 3.1 has no content. Perhaps, Subsection 3.1 was meant to be Section 4, and $3.2->4.14$. $->4.24 .1->4.3$ and so on?

Interactive comment on Ann. Geophys. Discuss., https://doi.org/10.5194/angeo-2020-48, 2020. 